\title{
Etudes cytogénétiques de la fibrose cutanée humaine radioinduite
}

\author{
B. MARTINS ${ }^{* * *}$, L. SABATIER ${ }^{* *}$, R. CHAMORRO ${ }^{* \star}$, \\ M. RICOUL**, A. PINTON**, R. LUBIN**,

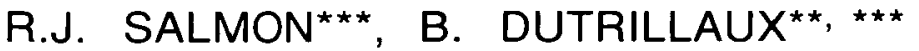 \\ *LNETI, Estrada Nacional n 10, 2685 Sacavém, Portugal ; \\ **Laboratoire de cytogénétique, Commissariat à l'énergie atomique, \\ DSV/DPTE, BP 6, 92265 Fontenay-aux-Roses Cedèx ; \\ ***Institut Curie, 26, Rue d'Ulm, 75231 Paris Cedex 05
}

Une étude cytogénétique in vivo de la fibrose cutanée radioinduite a été réalisée sur quinze personnes ayant subi une amputation du sein après rechute tumorale. Afin de comprendre l'origine de la fibrose, nous avons tenté de déterminer si ces comportements cellulaires anormaux pouvaient être corrélés à la présence d'anomalies chromosomiques. Vingt-deux cultures sous forme d'explants et six par digestion enzymatique ont été réalisées. Tous ces malades présentaient des caryotypes anormaux possédant plusieurs remaniements par cellule. Nous avons pu vérifier que douze cultures comportent un clone majoritaire et neuf autres un clone prédominant. Les clones observés ont en moyenne 2 à 11 points de cassure chromosomique. Nous avons pu conclure que : les fibroblastes responsables de la cicatrisation présentent de nombreux remaniements chromosomiques équilibrés, ils sont donc issus de tissus irradiés, il n'y a pas de processus intense de sélection clonale in vivo de la fibrose, il y a un avantage sélectif in vitro de certains clones.

La sélection in vitro des clones observés pourrait avoir été favorisée par la technique de culture sous forme d'explants. II nous a donc semblé indispensable d'étudier les caryotypes des cultures primaires de fibroses après digestion enzymatique afin de connaître la répartition in vivo des anomalies chromosomiques et leur devenir in vitro. 